\title{
A Workbench for Biomedical Applications Based on Image Analysis
}

\author{
Carlos Borau ${ }^{1}$, Cristina del Amo ${ }^{1}$, Jesús Asín ${ }^{2}$, Nieves Movilla ${ }^{1}$, \\ Mar Cóndor ${ }^{1}$, and José Manuel García-Aznar ${ }^{1}$ \\ ${ }^{1}$ Department of Mechanical Engineering, Aragón Institute of Engineering Research (I3A), \\ University of Zaragoza, Zaragoza, Spain \\ ${ }^{2}$ Department of Statistical Methods, University of Zaragoza, Zaragoza, Spain
}

\begin{abstract}
Unraveling the underlying mechanisms involved in single and collective cell migration, organization, tissue formation and regeneration are some of the currently spearheading research topics worldwide. To overcome the intrinsic difficulties associated to realistic environments in $3 \mathrm{D}$, a multi-disciplinary framework combining computer simulations and experiments is proposed. The success of this approach relies on result validation (quantita-tive comparison: computational vs. experimental), so we have polished different techniques and computational tools to get as accurate measurements as possible. For that, we take advantage of our own designed microfluidic devices and perform thorough image and statistical analyses.
\end{abstract}

Keywords: Image analysis $\cdot$ Microdevices $\cdot$ Biomedical application

\section{Introduction}

Image analysis has become a powerful tool in multiple biomedical applications, ranging from clinical cases to molecular/cellular experiments. The main purpose of this image processing is to extract relevant information or knowledge, by means of quantitative computing, which is very useful to perform comparative studies. Most of the experiments involving cell behavior have been performed in 2D due to its simplicity. However, 3D conditions are biologically more relevant, although they require much more complex analyses. Thanks to the latter advances in microfluidics, mimicking realistic environments is feasible through the fabrication of microdevices with multiple geometries, allowing an enormous flexibility and adaptability. Moreover, thanks to current technology, analysis tasks usually consuming weeks of manual work can be now performed in hours, or even minutes, by using proper image processing methods. Still, there are image analyses requiring a deep knowledge on the subject, especially those related to the underlying mechanics (e.g. strain, stress). To cope with these drawbacks, we have developed our own software to quantitatively analyze specific in-vitro tests (both 2D and 3D), using a wide variety of image analysis methods (e.g. thresholding, matrix convolution, skeletonization, machine learning etc.) and statistical tools (significance 
tests, regression models etc.). Specific applications involve for instance particle transport (quantification and analysis of bead trajectories and velocities) cell migration (MSD analysis, speeds, trajectories, directionality, diffusivity), angiogenic sprouting (quantification of length and frequency over time, 3D reconstruction), network alignment (anisotropic index of fibers), actin flow at the lamellipodium (velocity mapping, cyclic feature extraction) or dendritic protrusions (analysis of number, size, lifespan, subbranching) among others. Furthermore, this software is flexible and can be adapted and extended to a wide variety of in-vitro experiments. In this work, we describe the workbench that we have developed taking advantage of our own microfluidic devices and present via examples some of the already built in applications. We also discuss its strengths and weaknesses and finally we propose the possible future paths of this tool. Although we are still in a development stage, the expected outcome of our work is to eventually make such applications customizable and available to the market through an online service.

\section{Materials and Methods}

We fabricate our own microdevices following methodology described by Shin et al. [1]. Hence, soft lithography is employed to achieve positive SU8 $240 \mathrm{~lm}$-relief patterns of the desired geometry onto a silicon wafer (Stanford University). Polydimethylsiloxane (PDMS, Sylgard 184, Dow Corning $\mathrm{GmbH}$ ), mixed at a 10:1 ratio of base to curing

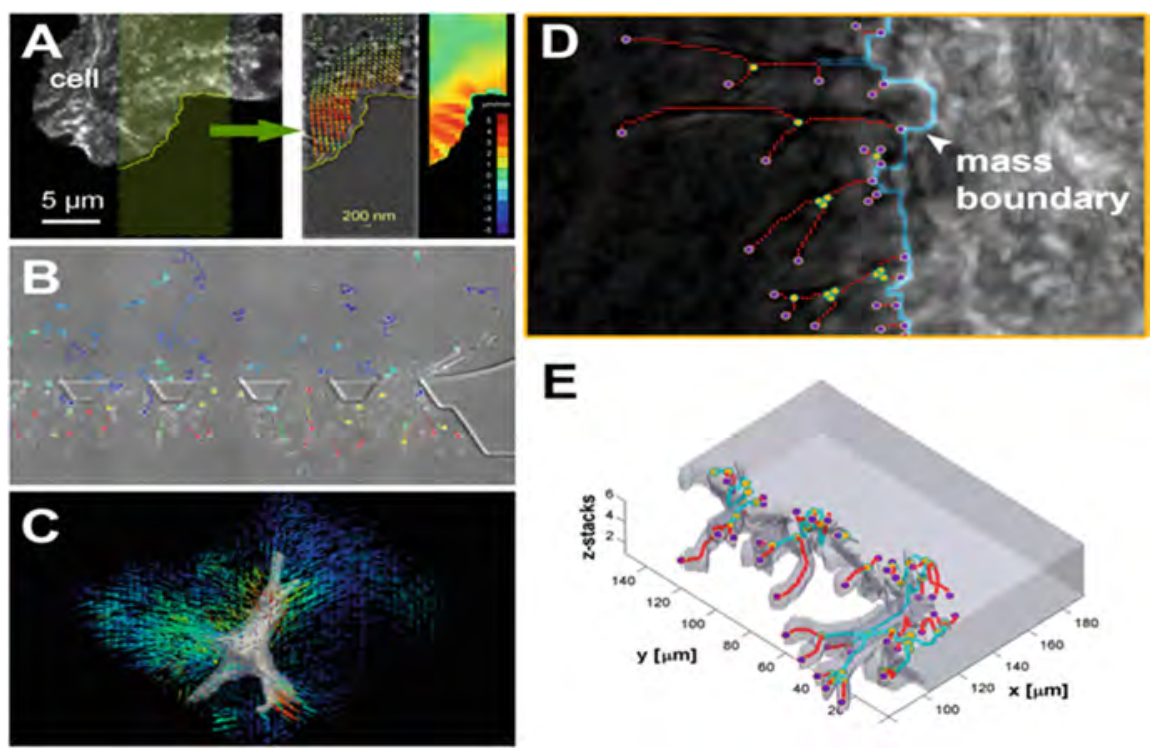

Fig. 1. Some examples of already built-in applications (A) Actin flow velocity mapping at the lamellipodium [2]. (B) Automated cell tracking in 2D [3, 4] (C) TFM reconstruction showing 3D extracellular matrix displacements around a cell. (D) Sprout detection in 2D [5]. (E) Sprout reconstruction in $3 \mathrm{D}[5]$. 
agent, is then poured and cured onto the SU8 master. The replica molded layer is trimmed, perforated and autoclaved. Finally, the PDMS devices and $35 \mathrm{~mm}$ glassbottom petri dishes (Ibidi) are plasma bonded and treated with poly-D-lysine (PDL) solution at $1 \mathrm{mg}$ mL21 (Sigma-Aldrich) for an enhanced surface-matrix attachment.

Image and statistical analysis are performed with hand-coded scripts using Matalb (Fig. 1).

\section{Discussion and Conclusions}

Nowadays, thousands of laboratories deal with the problem of proper image analysis, which can be really challenging specially when working on 3D in-vitro experiments. This kind of research involves training or hiring an image-analysis specialist, with the subsequent economical and time costs. Although there exist a wide variety of alternatives, the use of third party software is often expensive and non-straightforward, meaning that its functionality may be limited and not exactly oriented to your necessities. In fact, most of the available software only deals with the image processing, but not with the succeeding quantification of the data. For this reason, we have developed our own specific codes of image processing and post-analysis. This software allows not only to extract and analyze data from experiments in $2 \mathrm{D}$ and $3 \mathrm{D}$, including cell or particle tracking, shape detection, or sprouting measurement among others, but also to link those images to the underlying mechanics (e.g. displacement, strain or stress) through Finite Element Analysis (FEA). In fact, it has been successfully used so far to analyze and quantify chemotaxis, migratory patterns depending on mechanical stimuli and sprouting dynamics for different growth factors $[3,5,6]$. Offering these complete processing to the research community as a commercial online service would be of great interest for many laboratories that need to perform complex analyses and do not have the time, the experience or the resources to use other available tools. Furthermore, we plan to offer specialized quantitative analyses on-request, meaning that apart from a catalog of prebuilt functions, the potential client will be able to ask for specific analyses that will be, after a feasibility study, expressly developed and added to the catalog of services. In this way, our services directory will grow periodically and will be up to date with leading research analysis. Hence, as strengths and opportunities we can highlight our multidisciplinary engineering and experimental experience, the so far successful results of our software, its scalability and the large potential market currently available. Nonetheless, we have to take into account our lack of experience on the commercialization of services or possible threats such as the eventual emergence of other analysis-related companies.

To summarize, the innovation potential of our work lies on providing customizable image processing and data quantification services, including personalized reports, focused on but not limited to 3D cell cultures that will allow biomedical laboratories to easily externalize analysis tasks and therefore to focus on their own research activity.

Acknowledgments. This work is supported by the European Research Council (ERC) through project ERC-2012-StG 306571, the Spanish Ministry of Economy and Competitiveness (DPI2015-64221-C2-1-R) and the Government of Aragon (C126/2015). 


\section{References}

1. Shin, Y., Han, S., Jeon, J.S., Yamamoto, K., Zervantonakis, I.K., Sudo, R., Kamm, R.D., Chung, S.: Microfluidic assay for simultaneous culture of multiple cell types on surfaces or within hydrogels. Nat. Protoc. 7, 1247-1259 (2012)

2. Borau, C.: Multiscale computational modeling of single cell migration in 3D. Doctoral thesis (2013). http://zaguan.unizar.es/record/12585

3. Moreno-Arotzena, O., Borau, C., Movilla, N., Vicente-Manzanares, M., García-Aznar, J.M.: Fibroblast migration in $3 \mathrm{D}$ is controlled by haptotaxis in a non-musclemyosin II-dependent manner. Ann. Biomed. Eng. 12, 3025-3039 (2015)

4. Wu, P.-H., Giri, A., Wirtz, D.: Statistical analysis of cell migration in 3D using the anisotropic persistent random walk model. Nat. Protoc. 10, 517-527 (2015)

5. del Amo, C., Borau, C., Gutiérrez, R., Asín, J., García-Aznar, J.M.: Quantification of angiogenic sprouting under different growth factors in a mi-crofluidic platform. J. Biomech. 49(8), 1340-1346 (2015)

6. del Amo, C., Borau, C., Movilla, N., Asín, J., García-Aznar, J.M.: Quantifying 3D chemotaxis in microfluidic-based chips with step gradients of collagen hydrogel concentrations. Integr. Biol. 9(4), 339-349 (2017) 